\title{
A Survey on Molluscs Pests in Karnataka, India
}

\author{
Pinku Paul* and C.M. Rafee \\ Department of Agricultural Entomology University of Agricultural Sciences, \\ Dharwad, Karnataka, 580005, India \\ *Corresponding author
}

\begin{tabular}{|c|c|}
\hline & A B S T R A C T \\
\hline Keywords & $\begin{array}{l}\text { The present survey on molluscs pests was conducted at Dharwad and Haveri districts in } \\
\text { Karnataka from June } 2015 \text { to January } 2016 \text {. During that the maior snail noticed was giant }\end{array}$ \\
\hline $\begin{array}{l}\text { Giant African snail, } \\
\text { Slugs, Forest } \\
\text { nursery, } \\
\text { Horticulture } \\
\text { nursery. }\end{array}$ & $\begin{array}{l}\text { African snail (GAS), Achatina fulica Ferussac damaging both forest and horticultural } \\
\text { nurseries. The activity of snails in the forest and horticultural nurseries was started during } \\
24^{\text {th }} \text { standard week (June } 3^{\text {rd }} \text { week) of } 2015 \text { and persisted up to } 2^{\text {nd }} \text { standard week of } 2016 \\
\text { with peak infestation during } 41^{\text {st }} \text { standard week (Oct. } 2^{\text {nd }} \text { week) to } 45^{\text {th }} \text { standard week } \\
\text { (Nov. } 2^{\text {nd }} \text { week). The highest snail population was recorded in moringa nursery }\left(5.54 / 4 \mathrm{~m}^{2} \text { ) }\right.\end{array}$ \\
\hline Article Info & $\begin{array}{l}\text { followed by mango }\left(4.79 / 4 \mathrm{~m}^{2}\right) \text { in High-Tech Horticulture Unit }(\mathrm{HHU}) \text { of Dharwad. But in } \\
\text { other places the highest number of snail population observed on mango }\left(4.69 / 4 \mathrm{~m}^{2}\right)\end{array}$ \\
\hline $\begin{array}{l}\text { Accepted: } \\
28 \text { August } 2017 \\
\text { Available Online: } \\
10 \text { September } 2017\end{array}$ & $\begin{array}{l}\text { followed by papaya }\left(3.66 / 4 \mathrm{~m}^{2}\right) \text { and teak }\left(3.53 / 4 \mathrm{~m}^{2}\right) \text {. Whereas, the slug incidence was not } \\
\text { noticed during the study period. The incidence of snail was positively and significantly } \\
\text { correlated with rainfall, relative humidity and minimum temperature irrespective of the } \\
\text { nursery plants. }\end{array}$ \\
\hline
\end{tabular}

\section{Introduction}

Mollusca is the second largest phylum of the animal kingdom, forming a major part of the world fauna (Serb and Lydeard, 2003). The snails and slugs belong to the class Gastropoda and is the only class of molluscs which have successfully invaded land. Slugs are often described as snails without a shell, while snail bodies are enclosed in calcareous shells (Ramzy, 2009). Many species of land snails and slugs are among the important pests of agriculture, horticulture and garden crops in different parts of the world. Invasive species are recognized globally as a major threat to biodiversity and ecosystem health. Globally, about 35,000 species of land snails have been described and there may be 30,000 to 60,000 additional species yet to be described (Lydeard et al., 2004). Within modern India's boundaries 1129 species belonging to 140 genera and 26 families of land snails have been recorded (Ramakrishna et al., 2010).

Ravikumara et al., (2007) recorded the population of GAS throughout the year ranging from 1.00 to 91.25 snails $/ 10 \mathrm{~m}^{2}$ in arecanut at Shimoga district of Karnataka. Mallappa and Patil (2014) reported that population of giant African snail, A. fulica in betelvine garden in Belagutti village of Davanagere district occurred throughout the year ranging from 3.41 to 114.71 snails per 5 
$\mathrm{m}^{2}$ during 2011-12. The information regarding the incidence of molluscs pests in forest and horticulture nurseries are lacking. Hence, the study was undertaken with the following objective, "survey for snails and slugs infesting forest and horticulture nurseries".

\section{Materials and Methods}

Fixed plot survey was under taken in 10 locations of two districts (Dharwad and Haveri) in Karnataka from June, 2015 to January, 2016. During survey at each nursery the following observations were made on different types of seedlings/saplings.

No. of snails and slugs/ $4 \mathrm{~m}^{2}$ nursery saplings at 10 spots.

Foliar damage $(\%)$ caused by snails/slugs on 10 different saplings/seedlings.

Number of damaged leaves/ plant

Per cent leaf damage = -------------------- x 100

Total number of leaves/plant

\section{Places}

Hi-Tech Horticulture Unit, Dharwad; Forest Nursery (FN) MARS, Dharwad; FN, KC Park, Dharwad; FN, Sanjeevini Park; FN near kelager; FN, Gungargatti; FN, Sirsi; ARS, Malagi; ZHRS, Kumbhapur and medical/ aromatic nursery- Saidpur Farm.

\section{Correlation studies with weather parameter}

The fixed plot survey data on the incidence of GAS/slugs on different nurseries were subjected to correlation to know the relationship between pest incidence and weather parameters prevailed in Dharwad by using SPSS (Statistical Package for Social Sciences) software.

\section{Collection, identification of snail and slug species}

Snails and slugs were collected from the infested fields/nurseries from various localities and brought to the laboratory, reared in the cages and specimens were preserved in 70 per cent alcohol.

Only the shells of the snails and entire slugs were sent for identification to Dr. Venkitesan, Scientist C, Zoological Survey of India, Southern Regional Centre, Chennai.

\section{Results and Discussion}

The fixed plot survey was made once in a week from June, 2015 to January, 2016 and results are presented in tables 1 and 2. During survey the mean number of snail population per $4 \mathrm{~m}^{2}$ field inside the nursery was recorded on different saplings like mango, papaya, jamun, curry leaf, moringa, silver oak, teak, neem, pongamia, citrus and guava.

The incidence of snails and their damage on seedlings was observed from $24^{\text {rd }}$ standard week on different saplings grown in forest and horticultural nurseries of Dharwad district.

\section{Survey at Hi-Tech Horticulture Unit (HHU), UAS, Dharwad}

The snail population and per cent leaf damage was recorded on different forest/ horticulture seedlings at HHU, Dharwad from $24^{\text {th }}$ standard week to $4^{\text {th }}$ standered week is presented in the table 1. Among the different nurseries surveyed at HHU during 2015, the mean population of snails varied from 2.01 to 5.54 per $4 \mathrm{~m}^{2}$.

The higher snail population was recorded in moringa $\left(5.54 / 4 \mathrm{~m}^{2}\right)$ followed by mango $\left(4.79 / 4 \mathrm{~m}^{2}\right)$, jamun $\left(2.40 / 4 \mathrm{~m}^{2}\right)$ and lower 
snail population observed in curry leaf (2.01/4 $\mathrm{m}^{2}$ ). Whereas, maximum per cent leaf damage observed in moringa $(23.35 \%)$ followed by mango (12.89\%), curry leaf $(10.25 \%)$ and minimum in jamun $(7.27 \%)$.

\section{On moringa}

The incidence of $A$. fulica on moringa (4-5 months) revealed, that the first appearance of snail was noticed during $24^{\text {th }}$ standard week $\left(2.40 / 4 \mathrm{~m}^{2}\right)$ and persisted in nursery up to $51^{\text {st }}$ standard weeks (Dec. $4^{\text {th }}$ week).

Population density of snail found inside the nursery was ranged from 0.00 to 13.70 per $4 \mathrm{~m}^{2}$ with a mean population of $5.54 / 4 \mathrm{~m}^{2}$. Whereas, the maximum snail damage $(55.82 \%)$ was at $42^{\text {nd }}$ standard week. The mean per cent leaf damage inflicted by snail on moringa nursery was 23.35 per cent.

\section{On mango}

The maximum snail population on mango nursery $\left(12.90 / 4 \mathrm{~m}^{2}\right)$ was recorded more during $37^{\text {th }}$ standard week with average population of 4.79 per $4 \mathrm{~m}^{2}$.

The maximum leaf damage of 27.32 per cent was recorded at $44^{\text {th }}$ std week with a mean leaf damage of 12.89 per cent by the giant African snail on mango nursery.

\section{On jamun}

The first appearance of snails was started during $24^{\text {th }}$ standard week and persisted in nursery up to $46^{\text {th }}$ standard week.

The population density on jamun seedlings varied from 0.00 to 6.90 per $4 \mathrm{~m}^{2}$ with the mean population 2.40. Maximum leaf damage of was recorded 21.82 per cent at $41^{\text {st }}$ std week. The mean leaf damage caused by the giant African snail on jamun nursery was 7.27 per cent.

\section{Curry leaf}

The first appearance of snails was noticed during $25^{\text {th }}$ standard week and persisted in nursery up to $49^{\text {th }}$ standard week. The average population ranged from 0.00 to 6.30 per $4 \mathrm{~m}^{2}$ with a seasonal mean of $2.01 / 4 \mathrm{~m}^{2}$.

Maximum leaf damage of 30.56 per cent was recorded at $42^{\text {nd }}$ std week with average of 10.25 per cent. After $52^{\text {nd }}$ std week onwards there was no leaf damage.

\section{Survey in forest Nursery, MARS, Dharwad}

\section{On mango}

The incidence of A. fulica on mango nursery revealed that, the first appearance of snails was noticed during $24^{\text {th }}$ standard week and persisted up to $49^{\text {th }}$ standard week in nursery.

The snail population inside the field was ranged from 0.00 to 11.40 per $4 \mathrm{~m}^{2}$ with average of $4.69 / 4 \mathrm{~m}^{2}$.

Whereas, the maximum damage of 35.10 per cent was recorded in $41^{\text {st }}$ standard week which coincided with the highest snail population $\left(11.40 / 4 \mathrm{~m}^{2}\right)$.

The mean leaf damage by the population of snail on mango nursery was 12.29 per cent and no damage was found after $49^{\text {th }}$ std week (Table 2).

\section{On silver oak}

The population density of giant African snail in silver oak nursery ranged from 0.00 to $5.20 / 4 \mathrm{~m}^{2}$ with mean of $2.15 / 4 \mathrm{~m}^{2}$. The first appearance of snails was noticed during $25^{\text {th }}$ standard week and persisted in nursery up to $50^{\text {th }}$ standard week in silver oak nursery. The maximum leaf damage (13.55\%) was recorded at $45^{\text {th }}$ std week with seasonal mean damage of 4.95 per cent. 
Table.1 Incidence of Achatina fulica on different crops in Hi-Tech Horticulture Unit, Dharwad

\begin{tabular}{|c|c|c|c|c|c|c|c|c|c|}
\hline \multirow{3}{*}{ Period } & \multirow{3}{*}{$\begin{array}{c}\text { SM } \\
\mathbf{W}\end{array}$} & \multicolumn{8}{|c|}{ Hi-Tech Horticulture Unit } \\
\hline & & \multicolumn{2}{|c|}{ Moringa (4 to 5 months) } & \multicolumn{2}{|c|}{ Mango (6 to 8 months) } & \multicolumn{2}{|c|}{ Jamun (10 to12 months) } & \multicolumn{2}{|c|}{ Curry leaf (6 to 8 months) } \\
\hline & & $\begin{array}{c}\text { No. of } \\
\text { snails } / 4 m^{2}\end{array}$ & Leaf damage (\%) & $\begin{array}{c}\text { No. of } \\
\text { snails/4m }\end{array}$ & Leaf damage (\%) & No. of snails $/ 4 \mathrm{~m}^{2}$ & Leaf damage (\%) & No. of snails $/ 4 \mathrm{~m}^{2}$ & Leaf damage (\%) \\
\hline June $3^{\text {rd }}$ week & 24 & 2.40 & 3.80 & 4.50 & 3.43 & 1.50 & 2.23 & 0.00 & 0.00 \\
\hline June $4^{\text {th }}$ week & 25 & 5.40 & 6.65 & 6.20 & 4.32 & 3.30 & 2.50 & 0.60 & 0.00 \\
\hline June $5^{\text {th }}$ week & 26 & 5.80 & 8.93 & 3.60 & 5.48 & 1.60 & 2.76 & 1.20 & 3.50 \\
\hline July $1^{\text {st }}$ week & 27 & 4.20 & 11.85 & 3.10 & 5.90 & 1.50 & 2.85 & 0.80 & 4.20 \\
\hline July $2^{\text {nd }}$ week & 28 & 3.80 & 13.16 & 2.90 & 6.52 & 2.10 & 3.30 & 0.60 & 5.43 \\
\hline July $3^{\text {rd }}$ week & 29 & 3.40 & 16.33 & 3.80 & 6.68 & 2.40 & 4.35 & 1.30 & 6.85 \\
\hline July $4^{\text {th }}$ week & 30 & 6.50 & 18.67 & 2.90 & 7.30 & 3.50 & 5.50 & 2.20 & 6.97 \\
\hline Aug. $1^{\text {st }}$ week & 31 & 7.20 & 20.17 & 4.50 & 8.56 & 2.60 & 7.68 & 3.40 & 7.68 \\
\hline Aug. $2^{\text {nd }}$ week & 32 & 8.40 & 25.60 & 6.90 & 8.95 & 3.10 & 8.85 & 4.70 & 9.75 \\
\hline Aug. $3^{\text {rd }}$ week & 33 & 9.800 & 28.55 & 4.50 & 9.05 & 3.80 & 9.06 & 3.90 & 10.89 \\
\hline Aug. $4^{\text {th }}$ week & 34 & 5.30 & 30.50 & 4.20 & 12.20 & 4.10 & 11.08 & 2.10 & 11.10 \\
\hline Sept. $1^{\text {st }}$ week & 35 & 8.50 & 34.85 & 5.40 & 13.35 & 3.70 & 11.15 & 3.30 & 13.32 \\
\hline Sept. $2^{\text {nd }}$ week & 36 & 10.20 & 37.16 & 3.70 & 15.86 & 4.50 & 11.96 & 2.90 & 14.65 \\
\hline Sept. $3^{\text {rd }}$ week & 37 & 8.70 & 39.32 & 12.90 & 16.50 & 4.60 & 13.35 & 5.60 & 15.98 \\
\hline
\end{tabular}

SMW-Standard Meteorological Weeks

Table.1 Contd.

\begin{tabular}{|c|c|c|c|c|c|c|c|c|c|}
\hline \multirow{2}{*}{ Period } & \multirow{2}{*}{$\begin{array}{c}\text { SM } \\
\text { W }\end{array}$} & \multicolumn{2}{|c|}{ Moringa (4 to 5 months) } & \multicolumn{2}{|c|}{ Mango (6 to 8 months) } & \multicolumn{2}{|c|}{ Jamun (10 to12 months) } & \multicolumn{2}{|c|}{ Curry leaf (6 to 8 months) } \\
\hline & & No. of snails/4m & Leaf damage $(\%)$ & No. of snails $/ 4 \mathrm{~m}^{2}$ & Leaf damage (\%) & No. of snails $/ \mathbf{4 m}^{2}$ & Leaf damage $(\%)$ & No. of snails $/ 4 \mathrm{~m}^{2}$ & Leaf damage (\%) \\
\hline Sept. $4^{\text {th }}$ week & 38 & 12.30 & 43.76 & 11.40 & 16.80 & 5.50 & 16.66 & 5.50 & 17.05 \\
\hline Sept. $5^{\text {th }}$ week & 39 & 6.60 & 45.25 & 6.70 & 17.30 & 3.10 & 16.85 & 3.10 & 22.08 \\
\hline Oct. $1^{\text {st }}$ week & 40 & 13.70 & 48.85 & 12.10 & 18.63 & 6.70 & 18.38 & 6.30 & 25.15 \\
\hline Oct. $2^{\text {nd }}$ week & 41 & 12.70 & 49.60 & 9.60 & 19.85 & 6.90 & 21.82 & 5.70 & 28.32 \\
\hline Oct. $3^{\text {rd }}$ week & 42 & 9.50 & 55.82 & 7.50 & 20.96 & 5.10 & 19.02 & 3.30 & 30.56 \\
\hline Oct. $4^{\text {th }}$ week & 43 & 7.20 & 52.30 & 6.10 & 24.60 & 4.20 & 17.13 & 2.20 & 26.63 \\
\hline Nov. $1^{\text {st }}$ week & 44 & 11.50 & 45.00 & 5.80 & 27.32 & 3.20 & 14.50 & 2.70 & 22.92 \\
\hline Nov. $2^{\text {nd }}$ week & 45 & 7.60 & 35.45 & 5.20 & 23.30 & 1.50 & 11.65 & 2.20 & 18.75 \\
\hline Nov. $3^{\text {rd }}$ week & 46 & 3.20 & 29.02 & 4.70 & 22.46 & 0.60 & 7.14 & 0.60 & 13.08 \\
\hline Nov. $4^{\text {th }}$ week & 47 & 4.90 & 24.17 & 5.10 & 19.56 & 0.00 & 0.00 & 1.30 & 9.50 \\
\hline Dec. $1^{\text {st }}$ week & 48 & 2.40 & 17.33 & 4.40 & 17.52 & 0.00 & 0.00 & 0.50 & 7.44 \\
\hline Dec. $2^{\text {nd }}$ week & 49 & 1.20 & 13.50 & 3.90 & 14.16 & 0.00 & 0.00 & 0.20 & 6.53 \\
\hline Dec. $3^{\text {rd }}$ week & 50 & 00 & 9.05 & 2.70 & 11.25 & 0.00 & 0.00 & 0.00 & 0.00 \\
\hline Dec. $4^{\text {th }}$ week & 51 & 0.40 & 5.86 & 1.50 & 8.63 & 0.00 & 0.00 & 0.00 & 0.00 \\
\hline Dec. $5^{\text {th }}$ week & 52 & 0.00 & 0.00 & 1.10 & 7.50 & 0.00 & 0.00 & 0.00 & 0.00 \\
\hline Jan. $1^{\text {st }}$ week & 1 & 0.00 & 0.00 & 0.70 & 3.58 & 0.00 & 0.00 & 0.00 & 00 \\
\hline Jan. $2^{\text {nd }}$ week & 2 & 0.00 & 0.00 & 0.50 & 2.10 & 0.00 & 0.00 & 0.00 & 00 \\
\hline Jan. $3^{\text {rd }}$ week & 3 & 0.00 & 0.00 & 0.00 & 0.00 & 0.00 & 0.00 & 0.00 & 00 \\
\hline Jan. $4^{\text {th }}$ week & 4 & 0.00 & 0.00 & 0.00 & 0.00 & 0.00 & 0.00 & 0.00 & 00 \\
\hline Mean & & 5.54 & 23.35 & 4.79 & 12.89 & 2.40 & 7.27 & 2.01 & 10.25 \\
\hline
\end{tabular}


Table.2 Incidence of Achatina fulica forest/horticulture seedlings in different forest nurseries (FN) located in and Dharwad (2015-16)

\begin{tabular}{|c|c|c|c|c|c|c|c|c|c|c|c|c|c|}
\hline \multirow{3}{*}{ Period } & \multirow{3}{*}{$\begin{array}{l}\mathbf{S} \\
\mathbf{M} \\
\mathbf{W}\end{array}$} & \multicolumn{4}{|c|}{ FN, MARS, Dharwad } & \multicolumn{4}{|c|}{ FN, KC Park } & \multicolumn{4}{|c|}{ FN, Sanjeevini Park } \\
\hline & & \multicolumn{2}{|c|}{$\begin{array}{c}\text { Mango (4-5 } \\
\text { months) }\end{array}$} & \multicolumn{2}{|c|}{$\begin{array}{c}\text { Silver oak (10- } \\
11 \text { months) } \\
\end{array}$} & \multicolumn{2}{|c|}{$\begin{array}{c}\text { Teak (9- } 10 \\
\text { months) }\end{array}$} & \multicolumn{2}{|c|}{ Neem (1 year) } & \multicolumn{2}{|c|}{$\begin{array}{l}\text { Papaya (4- } 5 \\
\text { months) }\end{array}$} & \multicolumn{2}{|c|}{$\begin{array}{c}\text { Teak (5-6 } \\
\text { months) }\end{array}$} \\
\hline & & $\begin{array}{c}\text { Snails/ } \\
4 \mathrm{~m}^{2}\end{array}$ & $\begin{array}{c}\text { Leaf } \\
\text { damage } \\
(\%)\end{array}$ & $\begin{array}{l}\text { Snail } \\
\mathrm{s} / 4 \mathrm{~m}^{2}\end{array}$ & $\begin{array}{c}\text { Leaf } \\
\text { damage } \\
(\%)\end{array}$ & $\begin{array}{l}\text { Snail } \\
\mathrm{s} / 4 \mathrm{~m}^{2}\end{array}$ & $\begin{array}{c}\text { Leaf } \\
\text { damage } \\
(\%)\end{array}$ & $\begin{array}{c}\text { Snails/ } \\
4 \mathrm{~m}^{2}\end{array}$ & $\begin{array}{c}\text { Leaf } \\
\text { damage } \\
(\%)\end{array}$ & $\begin{array}{l}\text { Snails/ } \\
4 \mathbf{m}^{2}\end{array}$ & $\begin{array}{c}\text { Leaf } \\
\text { Damage } \\
(\%)\end{array}$ & $\begin{array}{c}\text { Snails/ } \\
4 \mathrm{~m}^{2}\end{array}$ & \begin{tabular}{|c|} 
Leaf \\
Damage \\
$(\%)$
\end{tabular} \\
\hline June $3^{\text {rd }}$ week & 24 & 1.40 & 2.50 & 0.00 & 0.00 & 0.00 & 0.00 & 0.00 & 0.00 & 0.40 & 0.75 & 1.8 & 2.56 \\
\hline June $4^{\text {th }}$ week & 25 & 2.40 & 3.19 & 1.80 & 1.04 & 0.00 & 0.00 & 0.50 & 0.00 & 3.90 & 3.5 & 2.4 & 3.45 \\
\hline June $5^{\text {th }}$ week & 26 & 3.80 & 4.20 & 1.50 & 1.65 & 0.00 & 0.00 & 1.20 & 0.60 & 2.50 & 5.68 & 2.8 & 4.90 \\
\hline July $1^{\text {st }}$ week & 27 & 5.20 & 5.33 & 1.30 & 1.86 & 1.10 & 1.50 & 0.40 & 0.70 & 1.20 & 5.8 & 3.5 & 5.85 \\
\hline July $2^{\text {nd }}$ week & 28 & 4.80 & 5.67 & 2.20 & 2.25 & 1.40 & 2.50 & 0.60 & 0.90 & 3.80 & 7.1 & 3.8 & 6.65 \\
\hline July $3^{\text {rd }}$ week & 29 & 3.40 & 7.10 & 2.40 & 2.57 & 1.20 & 2.80 & 0.00 & 1.20 & 2.50 & 8.5 & 4.2 & 7.85 \\
\hline July $4^{\text {th }}$ week & 30 & 5.50 & 7.50 & 3.60 & 2.75 & 1.40 & 3.20 & 0.90 & 1.60 & 3.10 & 10.4 & 3.7 & 8.52 \\
\hline Aug. $1^{\text {st }}$ week & 31 & 5.80 & 8.30 & 3.90 & 3.15 & 2.50 & 3.50 & 0.70 & 1.80 & 6.40 & 13.5 & 4.3 & 8.78 \\
\hline $\begin{array}{c}\text { Aug. } 2^{\text {nd }} \\
\text { week }\end{array}$ & 32 & 6.30 & 8.50 & 2.80 & 3.37 & 2.70 & 3.90 & 1.40 & 2.10 & 8.70 & 14.5 & 6.5 & 9.95 \\
\hline Aug. $3^{\text {rd }}$ week & 33 & 6.50 & 10.60 & 3.70 & 3.62 & 0.70 & 4.50 & 0.50 & 2.90 & 5.00 & 15.7 & 4.6 & 10.05 \\
\hline Aug. $4^{\text {th }}$ week & 34 & 7.20 & 13.30 & 4.20 & 3.50 & 0.60 & 4.80 & 0.40 & 3.20 & 5.30 & 16.64 & 4.3 & 12.45 \\
\hline Sept. $1^{\text {st }}$ week & 35 & 7.70 & 13.80 & 2.60 & 3.80 & 0.50 & 4.90 & 0.90 & 3.80 & 7.40 & 18.8 & 3.4 & 13.67 \\
\hline $\begin{array}{l}\text { Sept. } 2^{\text {nd }} \\
\text { week }\end{array}$ & 36 & 8.30 & 19.90 & 3.90 & 4.20 & 0.70 & 5.10 & 1.60 & 3.50 & 10.30 & 22.4 & 3.9 & 15.40 \\
\hline $\begin{array}{l}\text { Sept. } 3^{\text {rd }} \\
\text { week }\end{array}$ & 37 & 8.60 & 22.10 & 2.20 & 5.55 & 1.90 & 5.30 & 1.90 & 2.70 & 6.40 & 24.5 & 4.8 & 16.50 \\
\hline Sept. $4^{\text {th }}$ week & 38 & 9.30 & 25.30 & 4.10 & 6.90 & 2.30 & 5.60 & 1.10 & 2.15 & 9.50 & 26.85 & 4.3 & 17.50 \\
\hline Sept. $5^{\text {th }}$ week & 39 & 8.70 & 28.50 & 2.20 & 8.01 & 1.20 & 6.80 & 0.70 & 2.40 & 6.70 & 30.4 & 6.6 & 18.00 \\
\hline Oct. $1^{\text {st }}$ week & 40 & 10.80 & 31.80 & 5.20 & 9.50 & 3.50 & 6.90 & 1.80 & 3.50 & 12.90 & 35.7 & 7.5 & 19.36 \\
\hline Oct. $2^{\text {nd }}$ week & 41 & 11.40 & 35.10 & 4.20 & 10.20 & 4.10 & 7.80 & 2.10 & 4.30 & 9.40 & 37.2 & 8.2 & 20.56 \\
\hline Oct. $3^{\text {rd }}$ week & 42 & 9.50 & 33.50 & 3.80 & 10.64 & 1.50 & 9.60 & 1.70 & 2.90 & 6.20 & 36.2 & 9.6 & 25.85 \\
\hline
\end{tabular}

SMW-Standard Meteorological Weeks, FN: Forest Nursery 
Table.2 Contd.

\begin{tabular}{|c|c|c|c|c|c|c|c|c|c|c|c|c|c|}
\hline \multirow{3}{*}{ Period } & \multirow{3}{*}{$\begin{array}{c}\mathbf{S} \\
\mathbf{M} \\
\mathbf{W}\end{array}$} & \multicolumn{4}{|c|}{ FN, MARS, Dharwad } & \multicolumn{4}{|c|}{ FN, KC Park } & \multicolumn{4}{|c|}{ FN, Sanjeevini Park } \\
\hline & & \multicolumn{2}{|c|}{$\begin{array}{c}\text { Mango (4-5 } \\
\text { months) }\end{array}$} & \multicolumn{2}{|c|}{$\begin{array}{l}\text { Silver oak (10- } \\
11 \text { months) } \\
\end{array}$} & \multicolumn{2}{|c|}{$\begin{array}{l}\text { Teak (9- } 10 \\
\text { months) }\end{array}$} & \multicolumn{2}{|c|}{ Neem (1 year) } & \multicolumn{2}{|c|}{$\begin{array}{c}\text { Papaya (4- } 5 \\
\text { months) }\end{array}$} & \multicolumn{2}{|c|}{ Teak (5-6 months) } \\
\hline & & $\begin{array}{c}\text { Snails/ } \\
4 \mathrm{~m}^{2}\end{array}$ & $\begin{array}{c}\text { Leaf } \\
\text { damage } \\
(\%)\end{array}$ & $\begin{array}{c}\text { Snails } \\
/ 4 \mathrm{~m}^{2}\end{array}$ & $\begin{array}{c}\text { Leaf } \\
\text { damage } \\
(\%)\end{array}$ & $\begin{array}{c}\text { Snails } \\
/ 4 \mathrm{~m}^{2}\end{array}$ & $\begin{array}{c}\text { Leaf } \\
\text { damage } \\
(\%)\end{array}$ & $\begin{array}{c}\text { Snails/ } \\
4 \mathrm{~m}^{2}\end{array}$ & $\begin{array}{c}\text { Leaf } \\
\text { damage } \\
(\%)\end{array}$ & $\begin{array}{c}\text { Snails/ } \\
4 \mathrm{~m}^{2}\end{array}$ & $\begin{array}{c}\text { Leaf } \\
\text { damage } \\
(\%)\end{array}$ & $\begin{array}{c}\text { Snails/ } \\
4 \mathrm{~m}^{2}\end{array}$ & $\begin{array}{c}\text { Leaf } \\
\text { damage } \\
(\%)\end{array}$ \\
\hline Oct. $4^{\text {th }}$ week & 43 & 7.20 & 29.50 & 2.90 & 11.35 & 1.40 & 11.50 & 1.30 & 2.10 & 3.60 & 31.56 & 7.5 & 26.60 \\
\hline Nov. $1^{\text {st }}$ week & 44 & 5.50 & 25.65 & 3.20 & 11.45 & 0.80 & 10.13 & 0.60 & 1.40 & 2.40 & 28.03 & 5.8 & 27.32 \\
\hline Nov. $2^{\text {nd }}$ week & 45 & 4.60 & 21.30 & 2.30 & 13.55 & 0.50 & 8.30 & 0.00 & 0.00 & 1.30 & 25.05 & 4.4 & 24.30 \\
\hline Nov. $3^{\text {rd }}$ week & 46 & 3.20 & 15.20 & 1.90 & 10.67 & 0.40 & 7.74 & 0.00 & 0.00 & 1.20 & 19.02 & 3.2 & 22.46 \\
\hline Nov. $4^{\text {th }}$ week & 47 & 3.90 & 13.80 & 2.30 & 9.64 & 1.30 & 6.05 & 0.00 & 0.00 & 0.60 & 13.54 & 2.9 & 19.56 \\
\hline Dec. $1^{\text {st }}$ week & 48 & 2.40 & 8.01 & 1.40 & 7.57 & 0.50 & 3.95 & 0.00 & 0.00 & 0.00 & 0.00 & 1.8 & 17.52 \\
\hline Dec. $2^{\text {nd }}$ week & 49 & 1.20 & 6.16 & 0.90 & 7.45 & 0.00 & 0.00 & 0.00 & 0.00 & 0.00 & 0.00 & 0.9 & 14.16 \\
\hline Dec. $3^{\text {rd }}$ week & 50 & 0.00 & 0.00 & 0.40 & 6.83 & 0.00 & 0.00 & 0.00 & 0.00 & 0.00 & 0.00 & 0 & 0.00 \\
\hline Dec. $4^{\text {th }}$ week & 51 & 0.00 & 0.00 & 0.00 & 0.00 & 0.00 & 0.00 & 0.00 & 0.00 & 0.00 & 0.00 & 0 & 0.00 \\
\hline Dec. $5^{\text {th }}$ week & 52 & 0.00 & 0.00 & 0.00 & 0.00 & 0.00 & 0.00 & 0.00 & 0.00 & 0.00 & 0.00 & 0 & 0.00 \\
\hline Jan. $1^{\text {st }}$ week & 1 & 0.00 & 0.00 & 0.00 & 0.00 & 0.00 & 0.00 & 0.00 & 0.00 & 0.00 & 0.00 & 0 & 0.00 \\
\hline Jan. $2^{\text {nd }}$ week & 2 & 0.00 & 0.00 & 0.00 & 0.00 & 0.00 & 0.00 & 0.00 & 0.00 & 0.00 & 0.00 & 0 & 0.00 \\
\hline Jan. $3^{\text {rd }}$ week & 3 & 0.00 & 0.00 & 0.00 & 0.00 & 0.00 & 0.00 & 0.00 & 0.00 & 0.00 & 0.00 & 0 & 0.00 \\
\hline Jan. $4^{\text {th }}$ week & 4 & 0.00 & 0.00 & 0.00 & 0.00 & 0.00 & 0.00 & 0.00 & 0.00 & 0.00 & 0.00 & 0 & 0.00 \\
\hline Mean & & 4.69 & 12.29 & 2.15 & 4.95 & 0.97 & 3.83 & 0.62 & 1.33 & 3.66 & 13.68 & 3.53 & 11.51 \\
\hline
\end{tabular}

Table.3 Correlation coefficient between snail population and weather parameters on different saplings

\begin{tabular}{|c|c|c|c|c|c|c|c|c|}
\hline Parameters & $\begin{array}{c}\text { Moringa } \\
\text { (HHU) }\end{array}$ & Mango & Jamun & Curry Leaf & Silver oak & Teak (KC park) & Neem & Papaya \\
\hline Max. Temp. & -0.022 & -0.004 & -0.010 & -0.013 & -0.027 & -0.028 & -0.013 & -0.017 \\
\hline Min. Temp. & $0.654 * *$ & $0.547 * *$ & $0.635 * *$ & $0.514 * *$ & $0.653 * *$ & $0.466 * *$ & $0.514 * *$ & $0.586 * *$ \\
\hline $\mathbf{R H}$ & $0.669 * *$ & $0.665^{* *} *$ & $0.578 * *$ & $0.604 * *$ & $0.647 * *$ & $0.504 * *$ & $0.481 * *$ & $0.616 * *$ \\
\hline Rain fall & $0.457 * *$ & $0.481 * *$ & $0.454 * *$ & $0.441 *$ & $0.452 * *$ & $0.461 * *$ & $0.380 *$ & $0.544^{* * *}$ \\
\hline
\end{tabular}

** Significant at 0.01 level (2-tailed)

*. Significant at 0.05 level (2-tailed) 
Table.4 Meteorological data recorded for the year 2015-16 at main Agricultural Research Station, Dharwad (June 2015 to January, 2016)

\begin{tabular}{|c|c|c|c|c|c|}
\hline Months & $\begin{array}{c}\text { Standard Meteorological } \\
\text { Weeks (SMW) }\end{array}$ & $\begin{array}{c}\text { Max } \\
\text { Temp. }\left({ }^{\circ} \mathrm{C}\right)\end{array}$ & $\begin{array}{c}\text { Min } \\
\text { Temp. }\left({ }^{\circ} \mathrm{C}\right)\end{array}$ & $\begin{array}{l}\text { Humidity } \\
(\%)\end{array}$ & $\begin{array}{l}\text { Total Rainfall } \\
\text { (mm) }\end{array}$ \\
\hline June $3^{\text {rd }}$ week & 24 & 28.4 & 20.9 & 67.9 & 4 \\
\hline June $4^{\text {th }}$ week & 25 & 26.1 & 21 & 87.1 & 51 \\
\hline June $5^{\text {th }}$ week & 26 & 28 & 21.5 & 82.5 & 22.2 \\
\hline July $1^{\text {st }}$ week & 27 & 29.2 & 20.9 & 77.9 & 12.6 \\
\hline July $2^{\text {nd }}$ week & 28 & 29.6 & 22.2 & 78.1 & 7.6 \\
\hline July $3^{\text {rd }}$ week & 29 & 28.8 & 21.3 & 76.6 & 4.8 \\
\hline July $4^{\text {th }}$ week & 30 & 27.7 & 20.7 & 76.8 & 8.6 \\
\hline Aug. $1^{\text {st }}$ week & 31 & 28.4 & 20.4 & 82.1 & 27.7 \\
\hline Aug. $2^{\text {nd }}$ week & 32 & 27.7 & 20.8 & 85.1 & 4.2 \\
\hline Aug. $3^{\text {rd }}$ week & 33 & 29 & 20.7 & 78 & 2.8 \\
\hline Aug. $4^{\text {th }}$ week & 34 & 29.9 & 20.6 & 77.3 & 6.2 \\
\hline Sept. $1^{\text {st }}$ week & 35 & 28.6 & 20.3 & 86.1 & 14 \\
\hline Sept. $2^{\text {nd }}$ week & 36 & 30.1 & 20.4 & 83.5 & 17.8 \\
\hline Sept. $3^{\text {rd }}$ week & 37 & 28.4 & 20.6 & 92.4 & 4.2 \\
\hline Sept. $4^{\text {th }}$ week & 38 & 28.8 & 20.6 & 86.9 & 28.4 \\
\hline Sept. $5^{\text {th }}$ week & 39 & 36.7 & 20.9 & 80.9 & 0.2 \\
\hline Oct. $1^{\text {st }}$ week & 40 & 29.7 & 20.4 & 95.3 & 165.8 \\
\hline Oct. $2^{\text {nd }}$ week & 41 & 30.2 & 20.5 & 81.6 & 11.6 \\
\hline Oct. $3^{\text {rd }}$ week & 42 & 32.4 & 19.3 & 51.4 & 0 \\
\hline Oct. $4^{\text {th }}$ week & 43 & 32.2 & 17.9 & 44.8 & 0 \\
\hline Nov. $1^{\text {st }}$ week & 44 & 30.9 & 20 & 79.5 & 7.6 \\
\hline Nov. $2^{\text {nd }}$ week & 45 & 30.8 & 18.3 & 68.9 & 0 \\
\hline Nov. $3^{\text {rd }}$ week & 46 & 30.3 & 17.9 & 71.1 & 0 \\
\hline Nov. $4^{\text {th }}$ week & 47 & 28.6 & 19.6 & 76.6 & 23.4 \\
\hline Dec. $1^{\text {st }}$ week & 48 & 30.6 & 16.8 & 67.3 & 0 \\
\hline Dec. $2^{\text {nd }}$ week & 49 & 29.4 & 14.6 & 59.5 & 0 \\
\hline Dec. $3^{\text {rd }}$ week & 50 & 31.7 & 18.1 & 66 & 0 \\
\hline Dec. $4^{\text {th }}$ week & 51 & 31.5 & 17 & 62.1 & 0 \\
\hline Dec. $5^{\text {th }}$ week & 52 & 29.8 & 13 & 44.2 & 0 \\
\hline Jan. $1^{\text {st }}$ week & 1 & 30.7 & 14.4 & 37.2 & 0 \\
\hline Jan. $2^{\text {nd }}$ week & 2 & 29.4 & 12.2 & 34.7 & 0 \\
\hline Jan. $3^{\text {rd }}$ week & 3 & 28.5 & 14.2 & 13.3 & 0.4 \\
\hline Jan. $4^{\text {th }}$ week & 4 & 30.2 & 15.9 & 14.2 & 0 \\
\hline
\end{tabular}




\section{In forest nursery near KC Park, Dharwad}

\section{On teak}

The average snail population in teak nursery ranged from 0.00 to 4.10 per $4 \mathrm{~m}^{2}$ with average of 0.97 snails per $4 \mathrm{~m}^{2}$ (Table 2).

The first incidence of snail was noticed at $27^{\text {th }}$ std week and it persisted up to $48^{\text {th }}$ std week. The maximum leaf damage was recorded at $43^{\text {rd }}$ std week i.e., 11.50 per cent with average damage of 3.83 per cent.

\section{On neem}

From table 2 it is clear that, during $41^{\text {st }}$ std week the mean maximum number of snails were recorded $2.10 / 4 \mathrm{~m}^{2}$. The mean snail population was ranged from 0.00 to 2.10 per 4 $\mathrm{m}^{2}$ nursery with mean of $0.62 / 4 \mathrm{~m}^{2}$.

The average leaf damage from June 2015 to January 2016 was 1.33 per cent per $4 \mathrm{~m}^{2}$ inside the neem nursery. While during $41^{\text {st }}$ std week, the maximum damage was 4.30 per cent which coincided with the highest snail population.

\section{In forest nursery at Sanjeevini Park, Dharwad}

\section{On papaya}

The incidence of A. fulica on papaya revealed that, the first appearance of snails was noticed during $24^{\text {th }}$ standard week and persisted in nursery up to $47^{\text {th }}$ standard week.

The snail population inside the nursery was ranged from 0.00 to 12.90 per $4 \mathrm{~m}^{2}$ with average of 3.66. Whereas, the maximum leaf damage of snails was (37.20\%) was noticed in $41^{\text {st }}$ standard week. The mean leaf damage by the population of snail on papaya nursery was 13.68 per cent.

\section{On teak}

The activity of snails on teak nursery was recorded from $24^{\text {th }}$ std week to $49^{\text {th }}$ std week. The average population ranges from 0.00 to 9.60 per $4 \mathrm{~m}^{2}$ with a seasonal mean of $3.53 / 4 \mathrm{~m}^{2}$. The highest leaf damage of 27.32 per cent was recorded at $44^{\text {nd }}$ std week with average of 11.51 per cent.

\section{Other places}

Survey was also conducted in other nurseries like forest nurseries near kelageri; forest nurseries of Gungargatti; forest nurseries of Sirsi; ARS, Malagi; ZHRS, Kumbhapur; medical/aromatic nursery- Saidpur Farm on different seedlings viz. guava, papaya, pongamia, Myristica sp., Beilschmiedia sp. and citrus. But there was no infestation of snails during kharif, 2015. Similarly, the incidence of slugs was also not noticed in different nurseries surveyed during June to January, 2015-16.

\section{Correlation Co-efficient between population of snails and weather parameters during kharif, 2015}

The data on population of snails collected in Dharwad was correlated with the prevailing climatic weather parameters the results are given in the table 3. Data revealed that, snail population was positive and significantly correlated with rainfall, relative humidity and maximum temperature irrespective of the nurseries where it was negatively correlated with maximum temperature.

\section{Collection and identification of snail and slug species}

Snails/ slugs were collected from the infested fields from various localities of Hangal and Ranebennur taluks of Haveri district and identified by Dr. Venkitesan, Sr. Scientist, 
Zoological Survey of India, Southern Regional Centre and Chennai. During the survey four species of snails viz., Allopeas gracile, Rachis punctatus, Macrochlamys pedina, Macrochlamys indica and three species of slugs viz., Pseudaustenia ater, Laevicaulis alte and Mariaella beddomei were collected from farmers field in Dharwad and Haveri district were identified from ZSI, Chennai.

The present investigation is in line with Basavaraju et al., (2000) who opined that the infestation of giant African snail on biofuel nursery, vegetable nursery, arecanut nursery and mulberry garden ranged from 8 to 15 per cent in Hassan district of Karnataka. The activity of giant African snail, A. fulica started during $24^{\text {th }}$ standard is an agreement with the work of Mallappa. Further, Rafee et al., (2013) stated that the number of snails recorded in different agriculture and horticultural crops was higher during September due to receipt of high rainfall. The present study damage to various nursery seedlings (1.33 to $23.35 \%)$ in close agreement with the studies of Mallappa (2014) who registered that three to four months old seedlings were severely damaged by giant African snail on forest nursery in Bealgavi district during August 2011.

Maximum temperature exerted negative and significant relationship, but highly significant and positive relationship was noticed with rainfall. Likewise, highly significant and positive correlation was with relative humidity. This finding corroborates with Mallappa (2014) who reported correlation between snail incidence and weather factor indicated that the highly significant and positive relationship with rainfall ( $\mathrm{r}$ $\left.=0.652^{* *}\right)$. During the year 1998 as high as $1006 \mathrm{~mm}$ of annual rainfall was received which was second highest in the century and thus recorded highest population of 257.2 snails/100 $\mathrm{m}^{2}$ area (Balikai, 2008). This corroborates with Thakur (2003) from Bihar who opined positive and significant relationship between snail population and relative humidity. On the basis of present investigation it is concluded that the infestation of giant African snail was found more on horticulture nurseries (moringa, papaya etc.) than the forest nurseries (silver oak, jamun etc). Rainfall and relative humidity are the important weather factor in population build-up of giant African snail in forest and horticulture ecosystem.

\section{Acknowledgement}

The authors are thankful to the workers of HiTech Horticulture Unit of UAS, Dharwad for their kind support during the experiment.

\section{References}

Balikai, R. A., 1999. Incidence of snail, Cryptozona semirugata (Beck) on some horticultural crops in Northern Karnataka. Pest Management in Horticultural Ecosystems. 5(1): 70-71.

Basavaraju, S. B., Kulapati, H., Gowda, C. C., Sharanappa, J., Murthy, N. K., and Hipparagi, K. J. S. 2000. Preliminary survey on the incidence of Achatina fulica Bowdich in Davanagere district. Current Research. 29(7-8):129.

Lydeard, C., Cowie, R. H., Ponder, W. F., Bogan, A. E., Bouchet, P., and Clark, S. A. 2004. The global decline of nonmarine molluscs. Bio Science. 54:321330.

Mallappa, C., 2014. Crop los estimation due to giant Afgrican snail, Achatina fulica Ferussac at different phonological stages of groundnut during 2012-13 and 2013-14. Ph. D. Thesis, University of Agricultural. Sciences, Dharwad, Karnataka, India.

Mallappa, C., and Patil, R. K. 2014. 
Population dynamics of giant African snail, Achatina fulica Ferussac (Stylommataphora: Achatinidae) in betelvine ecosystem. Journal of Experimental Zoology. 17(1):285-288. Rafee, C. M., Patil, R. R., Goud, K. B., Patil, R. K., Krishna, N. L., and Patil, R. H. 2013 Survey for infestation of giant African snail in Hubli taluka. Paper presented in: $10^{\text {th }}$ National Symposium on Soil Biology and Ecology- Soil Biota and Social Insects for Sustainable Agriculture, University of Agricultural Sciences, Bengaluru, Karnataka, India. 19-21, p.155.

Ramakrishna, Mitra, S. C., and Dey, A. 2010. Annotated checklist of Indian land mollusc. Zoological Survey of India, Kolkata. p.359.

Ramzy, R. R., 2009. Biological and ecological studies on land snails at Assiut, Egypt. M. Sc. Thesis, Faculty of
Science, Agriculture University, Egypt. Ravikumara, N. M. I., Manjunath, M., and Pradeep, S. 2007. Seasonal incidence of giant African snail, Achatina fulica Bowdich (Gastropoda: Achatinidae) in areca ecosystem. Karnataka Journal of Agricultural Science. 20(1):157-158.

Serb, J. M., and Lydeard, C. 2003. Complete mtDNA sequence of the North American freshwater mussel, Lampsilis ornata (Unionidae): an examination of the evolution and phylogenetic utility of mitochondrial genome organization in Bivalvia (Mollusca). Molecular Biology and Evolution. 20:1854-1866.

Thakur, S., 2003. Population dynamics of giant African snail, Achatina fulica Bowdich (Stylommatophora: Achatinidae) in North Bihar. Journal of Applied Zoology and Research. 14:151154.

\section{How to cite this article:}

Pinku Paul and Rafee, C.M. 2017. A Survey on Molluscs Pests in Karnataka, India. Int.J.Curr.Microbiol.App.Sci. 6(9): 3123-3132. doi: https://doi.org/10.20546/ijcmas.2017.609.386 